\title{
Towards a Convergent Development of Learning Analytics
}

\author{
Xavier Ochoa, Arnon Hershkovitz, Alyssa Wise, Simon Knight \\ Editors, Journal of Learning Analytics \\ jla.editorial@gmail.com
}

\section{WHERE WE ARE NOW}

In the last 7 years, since the first LAK conference, Learning Analytics has grown rapidly as a field from a small group of interested scholars and practitioners to one of the most scientifically successful and institutionally accepted areas of Learning and Educational Technologies. Learning Analytics is often referred as a "Middle-Space" where experts from diverse fields (from the Learning Sciences, Computer Science, Human-Computer Interaction, Psychology and Behavioural Sciences, just to name a few) share their perspectives on how to better understand and optimize learning processes and environments using this new instrument called Data Science.

The multidisciplinary discussion has provided a rich background of paradigms, theories, methodologies and tools borrowed to craft Learning Analytics systems and conduct studies about how and under what conditions they are most effective. While important to a field in formation, this abundance of influences creates challenges that we should address while maintaining the multivocality of the field:

- Challenge 1: It is difficult to have rigorous yet fair review of scientific works. What is a common technique in Computer Science research may be uncommon or not well-understood in the Learning Sciences and vice-versa. Reviewers have generally been trained in only one (or maybe two) of the multiple disciplines on which Learning Analytics draws and thus may subject a work to overly exacting critique in some areas, while ignoring others. Soliciting diverse scholars provides comprehensive coverage, but often produces reviews that are difficult to reconcile. Specifically, reviewers (and editors) can have a hard time appreciating the full contribution of a work that is foreign to their original field.

- Challenge 2: Studies are rarely comparable. Even when applied to the same problem, for example drop-out prediction, it is very difficult to assess how much better one of two approaches (offered by different researchers using different data) are compared with one and another. The lack of common methodologies and indicators to assess the results of our algorithms and, more importantly, the impact that the system has on the learning process, makes it difficult to measure progress. 
(2017). Towards a convergent development of learning analytics. Journal of Learning Analytics, 4(3), 1-6. http://dx.doi.org/10.18608/jla.2017.43.1

- Challenge 3: Sub-optimal methodologies and tools are often applied when better alternatives exist. While it is not possible, or even desirable, to standardize research methodologies and tools, there are always best-practices shared among research communities. What are the recommended methodologies to evaluate the usability of an interface? What are the best statistical tests to assess the reliability of human evaluators? How to best measure the accuracy of a risk-prediction system? And the most dreaded one, how do we measure the impact of Learning Analytics tools? Currently, these questions receive different (usually irreconcilable) answers according to the background of the researcher. Furthermore, the choices made are not always up-to-par with the recommendations provided by the foundational field that focuses on these specific aspects.

Fortunately, there is a way to ease these issues without reducing the richness of Learning Analytics research: a voluntary convergence to a minimum set of common practices. This set of shared practices will enable studies about the same topic to be comparable; for example, a common evaluation indicator or metric can be identified and used among studies regardless of methodology and tools. This set of (evolving) best practices can also establish what is state-of-the-art and eliminate guesswork when selecting methodologies to solve or measure common problems or situations. The development of this common minimum language and standards will greatly help reviewers to better understand and evaluate the quality of contributions from lesser-known perspectives.

Developing a minimum set of common practices may seem to be a far-fetched and hard-to-implement goal, however, it is exactly what is already happening in several sub-fields of Learning Analytics, such as Reflective Writing and Cross and Multimodal Learning Analytics. Researchers working in these two subfields have regularly organized themselves around workshops and special journal issues, not only to showcase the state-of-the-art of their research but also to share methodologies, tools and even hardware designs. This sharing was born out of the relative complexity of these two endeavours as a way to avoid re-inventing the wheel in each subsequent study. These sub-fields are very close to developing their own minimum set of common practices. Far from precluding new practices, an established set of practices eliminates the need for discussion about what is already known to concentrate precisely on how it can be improved upon and expanded in the future. While the road to reach this goal will be different for different parts of the Learning Analytics field, these examples show that building the road is possible.

\section{HOW TO GET THERE}

Being an editor of the Journal of Learning Analytics (and for some of us, Program Committee chair of the Learning Analytics conference) provides one with a slightly different perspective on the field. We are able to read not only the papers that are accepted for publication but also those that are rejected and the reviewers' compliments and critiques that, in some way, represent the different voices in the 
(2017). Towards a convergent development of learning analytics. Journal of Learning Analytics, 4(3), 1-6. http://dx.doi.org/10.18608/jla.2017.43.1

community. We try to capitalize on this perspective to put forward a set of recommendations for a convergent development of the field of Learning Analytics:

- Recommendation 1: Develop a body of knowledge (and refer to an existing one). In order for a research community to build knowledge collectively, venue(s) for sharing information are needed. Moreover, these venues should exist in easy to disseminate formats that are accessible to all. Publishing research or practice papers in this (open-access) journal and LAK is a first step in developing the Learning Analytics body of knowledge, but it is not the only one. A further way to disseminate knowledge about Learning Analytics is to create compilations of current best practices, through handbooks (such as the recently released Handbook on Learning Analytics), training events (such as the annual SoLAR Learning Analytics Summer Institute) and ongoing educational opportunities (such as the upcoming series of EdX MicroMaster courses on Learning Analytics). More initiatives like these (both local and global) are needed to create a common ground for Learning Analytics research. The other side of the coin is the increased recognition of our studies within existing bodies of knowledge (and research communities) in the field of education (the "L" in LA). To this end we need state the the expected contribution of our studies to educational processes explicitly. In this way, Learning Analytics work will gradually become more accepted and considered in education at large. In addition, studies that are framed similarly (from an educational point of view) will be more easily comparable to each other (and might together highlight the contribution of different methodologies to a given area of study).

- Recommendation 2: Build sub-communities. Learning Analytics as a field is too big and diverse to be treated as a monolithic entity. However, we do see convergence at the more specific levels. All the major Learning Analytics initiatives provide procedures and venues to create and consolidate these sub-communities. For example, SoLAR, the Society for Learning Analytics research, supports the creation of Special Interest Groups (SIG) to discuss and set the standards for more particular aspects of Learning Analytics. The LAK conference is known for its openness to hosting workshops that do not revolve about papers, but around a table to discuss ways to improve specific aspects of the field. This journal encourages the submission of proposals to organize Special Sections about specific topics. These opportunities all serve as avenues to organize the current state-of-the-art and best practices produced by different sub-communities within the field of Learning Analytics.

- Recommendation 3: Train yourself through others. We should use the opportunities to evaluate works that come from different perspectives (by reviewing for JLA and LAK) with an open scientific/practitioner mind. We should also read others reviews about the same work in order to have a better appreciation the different points of view in the field. For example, LAK is implementing a meta-review model that will provide feedback to junior reviewers. Also, last year, LAK sent a basic reviewer report feedback with information about our reviewing practices 
(2017). Towards a convergent development of learning analytics. Journal of Learning Analytics, 4(3), 1-6. http://dx.doi.org/10.18608/jla.2017.43.1

compared with the rest of the community. Getting involved in "review-duty" is the best way to expand your scientific horizons and help to achieve coherence in the field. With the same spirit, JLA is introducing "Notes for Practice," a highlights section at the start of each research article where authors are encouraged to think about the possible use or implication of their research in the educational practice. (Conversely, practitioner articles will also now include "Notes for Research," a highlights section of the ways the learning analytics project conducted could lead to new research directions).

- Recommendation 4: Challenge the field. One proven and effective way to develop common evaluation standards is to organize research challenges where contributions are forced to be comparable with others. This can be achieved through a common dataset and evaluation metrics. These datasets and metrics do not need to be perfect (their use will immediately reflect their pros and cons) but can be a starting point to develop really good common indicators. While this can be organized through workshops at relevant conferences, this journal also offers the possibility to organize challenges through our new "Tools and Datasets" section. While these kinds of challenges have traditionally been technical in nature, Learning Analytics is a socio-technical field, suggesting that complementary challenges related to human aspects of learning analytics use (which might have somewhat different standards for evaluation) are also important to consider.

- Recommendation 5: Be diverse. Try to work in interdisciplinary teams. This is maybe the most difficult of the recommendations, but it is also the most important. True convergence in Learning Analytics will only be achieved when we understand the paradigms, research methodologies and tools used by the other fields involved. To be able to re-use the best that each field has to offer to understand and optimize learning, Learning Analytics work needs to employ as diverse a group of individuals as possible with different skills sets and points of view.

These recommendations should be transformed into specific initiatives in the field in order to be successful. Some of them are already taken place at different venues and levels. However, being aware of how these different elements come together to pave the road that is ahead (that is, moving towards a minimal set of common practices in Learning Analytics research and practice) helps us to make stronger process towards the next significant step in the development of the field.

\section{STARTING AT HOME (OR META-CONVERGENCE)}

Different Learning Analytics initiatives should also work in a coherent manner. For this reason, the Journal of Learning Analytics announces the following changes:

- Change 1: Double-Blind Review. Since the emergence of the field, the community has needed to balance the diversity of perspectives and approaches taken in the field, with a core fundamental 
(2017). Towards a convergent development of learning analytics. Journal of Learning Analytics, 4(3), 1-6. http://dx.doi.org/10.18608/jla.2017.43.1

commitment to high-quality research. As part of the development of the field, the LAK conference made the decision to migrate its research paper review process to a double-blind system. Following this example and reflecting a shared vision for the field, the Journal of Learning Analytics is also introducing a double-blind review process for all papers received starting January 2018.

- Change 2: Reviewer Feedback. Sharing the other (blinded) reviews of a paper with each reviewer has been practiced by many JLA editors for some time. Now, as a matter of policy, the Journal of Learning Analytics will make sure that each reviewer receives the reviews of other reviewers and the decision of the editor. This will help with cross-disciplinary understanding and reviewer calibration.

- Change 3: Editorial Board. To recognise the important contribution played by members of our community who provide their expertise in reviewing papers, the Journal of Learning Analytics will introduce a new editorial board. This board will support the editors in selecting reviewers for papers, making decisions on papers, and developing editorial policy for the journal. In the first instance, members will be selected from those members of the community who have contributed to the journal through high quality reviewing, with invitations to be sent out during 2018.

- Change 4: Publishing Code and (Sample) Data Alongside Papers. To improve the replicability of the studies published in the journal, we are encouraging papers in the following issues to publish, alongside the paper document, their code and sample data that help others to re-run the main analysis or experiments. The specifics about the platform and formats will be set out during the first half of 2018.

- Change 5: Less from Us, More from You. Starting in 2018, JLA will reduce the number of editorial to one per year. This will enable us to still communicate sufficiently about important issues for the journal and the field, but dedicate more of our efforts as editors to facilitating the development and publication of high quality learning analytics work.

These changes seek to homogenize the review process of JLA and LAK and to provide common publishing practices to all researchers and practitioners in Learning Analytics.

\section{FINALLY, AN EXAMPLE FROM THE CURRENT ISSUE}

In addition to a paper that presents a very interesting methodology for analysing student gaze, this issue of JLA includes a Special Section on Temporal Analysis. This can be seen as an effort to share the bestpractices on how to solve a specific problem (the analysis of data generated continuously through time) in the field of Learning Analytics. As you read the papers in this special section you will see the richness 
(2017). Towards a convergent development of learning analytics. Journal of Learning Analytics, 4(3), 1-6. http://dx.doi.org/10.18608/jla.2017.43.1

of different approaches all trying to meet a common goal. Diversity and innovation in our approaches to analysing learning data is what sets Learning Analytics apart from other fields and is also what has allowed us to grow as fast as we have done. Now is time to bring together this diversity in conversation that is convergent, but retains its multi-vocal character. We need to make the effort to both deeply understand and challenge the assumptions of work that comes from traditions other than our own. In this way, we start to build the set of common practices, language and standards of a mature field. 